\title{
ENERGY DEPENDENCE OF BEAM-BEAM INTERACTIONS AT LEP
}

\author{
H. Burkhardt, CERN, Geneva, Switzerland
}

\section{Abstract}

LEP has been operated for several years at beam energies around $45 \mathrm{GeV}$ and more recently at beam energies of 65 and $86 \mathrm{GeV}$, thus covering about a factor of two in beam energies.

Vertical beam-beam tune shifts exceeding 0.04 were reached at all energies.

At the lower energies, emittance increase using wigglers was used successfully to avoid excessive beam blow-up and flip-flop from beam-beam interactions and allowed to operate safely at the beam-beam limit throughout fills lasting typically 10 hours. Significant non-Gaussian tails were observed in the vertical plane and have limited the maximum current in collisions.

At the higher energies, the best performance in terms of luminosity and beam-beam tune shift demands higher currents per bunch and minimization of emittances. Vertical to horizontal emittance ratios below $0.5 \%$ have been achieved.

\section{INTRODUCTION}

The following quantities will be used:

$$
\begin{aligned}
e, m_{e}, r_{e} & : \text { electron charge, mass and classical radius } \\
f & : \text { revolution frequency, } 11245.5 \mathrm{~Hz} \\
i & : \text { bunch current } \\
E & : \text { beam energy }
\end{aligned}
$$

The interaction of the particles of one beam with the electromagnetic fields of the other produces a horizontal and vertical tune spread of:

$$
\begin{gathered}
\xi_{x}=\frac{r_{e} m_{e} i \beta_{x}^{*}}{2 \pi e f E \sigma_{x}^{2}\left(1+\frac{\left.\sigma_{y}\right)}{\left.\sigma_{x}\right)}\right.} \\
\xi_{y}=\frac{r_{e} m_{e} i \beta_{y}^{*}}{2 \pi e f E \sigma_{x} \sigma_{y}\left(1+\frac{\left.\sigma_{y}\right)}{\left.\sigma_{x}\right)}\right.}
\end{gathered}
$$

These formulas apply Gaussian shaped bunches, colliding head on. The quantities $\xi_{x}$ and $\xi_{y}$ are referred to as beambeam tune shift parameters. For flat beams $\left(\sigma_{y} \ll \sigma_{x}\right)$ we can write in good approximation:

$$
\begin{gathered}
\xi_{x}=\frac{r_{e} m_{e}}{2 \pi e f E} \cdot \frac{i \beta_{x}^{*}}{\sigma_{x}^{2}}=\frac{r_{e} m_{e}}{2 \pi e f E} \cdot \frac{i}{\varepsilon_{x}} \\
\xi_{y}=\frac{r_{e} m_{e}}{2 \pi e f E} \cdot \frac{i \beta_{y}^{*}}{\sigma_{x} \sigma_{y}}
\end{gathered}
$$

For the ratio of the tune shift parameters we obtain:

$$
\frac{\xi_{y}}{\xi_{x}}=\frac{\beta_{y}^{*}}{\beta_{x}^{*}} \frac{\sigma_{x}}{\sigma_{y}}=\sqrt{\frac{\beta_{y}^{*} \varepsilon_{x}}{\beta_{x}^{*} \varepsilon_{y}}}
$$

Horizontal and vertical beam-beam tune shift parameters are equal if the ratio of vertical to horizontal $\beta$ functions at the interaction point $\left(\beta_{y}^{*} / \beta_{x}^{*}\right)$ is equal to the emittance ratio $\kappa=\varepsilon_{y} / \varepsilon_{x}$. The luminosity per interaction region is :

$$
\mathcal{L}=\frac{i^{2} k}{4 \pi e^{2} f \sigma_{x} \sigma_{y}}=\frac{E i k \xi_{y}}{2 e r_{e} m_{e} \beta_{y}^{*}}
$$

For constant beam size, the beam-beam tune shift parameters increase linearly with current and the luminosity with current squared.

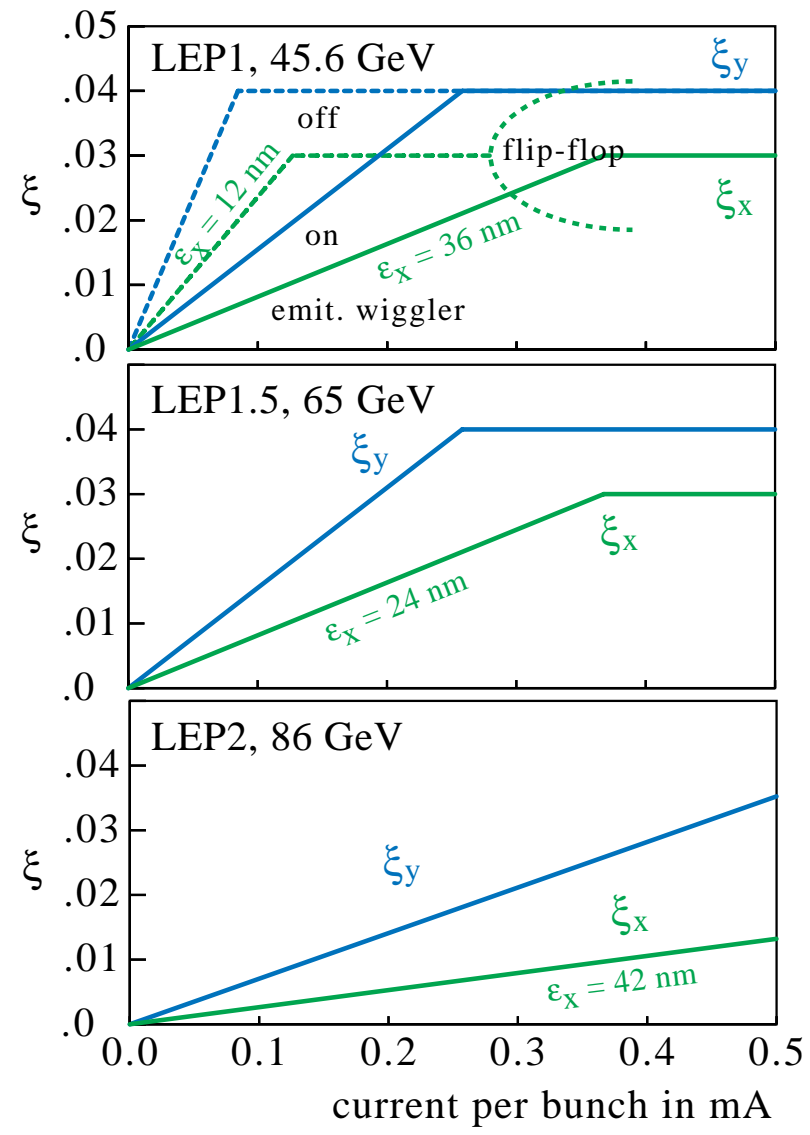

Figure 1: Illustration of the expected behavior of $\xi$ with current for $\beta_{x}^{*}=2.5 \mathrm{~m}, \beta_{y}^{*}=0.05 \mathrm{~m}$ and an emittance ratio $\kappa=0.5 \%$. The vertical beam-beam tune shift parameter was assumed to saturate at 0.04 and the horizontal beam beam tune shift parameter at 0.03 . 
For higher currents, the beam-beam interaction will lead to an increase in beam sizes and to saturation of the beambeam tune shift parameters at values of typically 0.04 for a well tuned machine. This is referred to as operation at the beam-beam limit: Emittances and luminosity increase linearly with current. The highest luminosity performance is obtained for operation at the beam-beam limit (at maximum $\xi$ ) throughout the whole fill. Fig. 1 illustrates the expected behavior of the beam-beam tune shift parameters as function of the bunch current.

For a given optics, the horizontal emittance scales with beam energy squared. $\xi_{x}$ and the damping time are proportional to $E^{-3}$. At LEP1, the beam-beam limit can be reached for very low currents $(0.1 \mathrm{~mA})$. To collide safely higher currents and to avoid uncontrolled unequal emittance blow-up (flip-flop), the horizontal emittance is increased using a wiggler in a dispersive region.

\section{LEP1, E=45.6 GEV}

LEP was operated in the years 1989 to 1995 at beam energies close to $45.6 \mathrm{GeV}$ for the production of Z-Bosons [1]. It took several years until beam-beam tune shifts exceeded values of 0.04 .

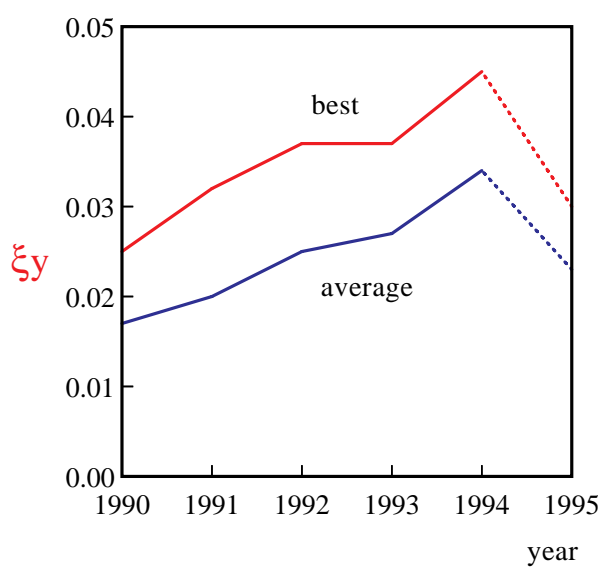

Figure 2: Best and average vertical beam-beam tune shift $\xi_{y}$ for 6 years of operation at LEP1 energies.

Fig. 2 shows the average and best vertical beam-beam tune shift parameters reached during the years of LEP1 operation. The best values with some comments on the operation are listed below:

1990: $\xi_{y}=.025$, odd integer tunes $71 / 77$ for low coupling, $60^{\circ}$ phase advance.

1991: $\xi_{y}=.032$, even integer tunes $70 / 76$ for improved beam-beam tune shift.

1992: $\xi_{y}=.037$, from $60^{\circ}$ to $90^{\circ}$ phase advance in both planes, realignment of LEP, emittance control.

1993: $\xi_{y}=.037,90^{\circ} / 60^{\circ}$ optics, from $4+4$ to $8+8$ bunches using the Pretzel scheme, introduction of golden orbits.
1994: $\xi_{y}=.045$, no change compared to 1993 , operational optimization based on golden orbits.

1995: $\xi_{y}=.030,12+12$ bunches using bunch trains and vertical separation.

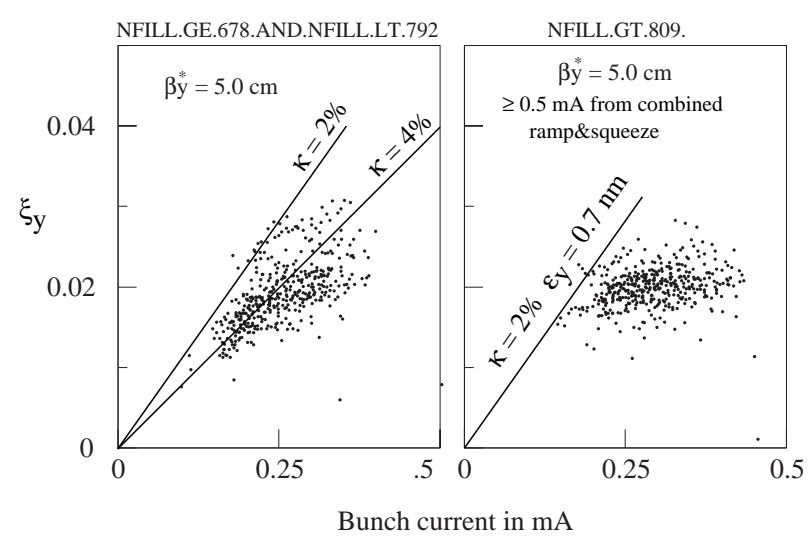

Figure 3: Vertical beam-beam tune shift parameter $\xi_{y}$ as function of bunch current as observed earlier (left) and later (right) in 1991.

In Fig. 3, it can be seen, that operation at LEP1 energies became only beam-beam limited later in 1991. The vertical beam-beam tune shift parameter started to saturate on average at values of about 0.02 . In 1992, the phase advance was increased to $90^{\circ}$, resulting in $12 \mathrm{~nm}$ horizontal emittance (compared to $35 \mathrm{~nm}$ with $60^{\circ}$ ). Using a wiggler in a dispersive region, the horizontal emittance could be adjusted between 12 and $36 \mathrm{~nm}$. This was very successful and allowed stable operation with $\xi_{y}$ regularly exceeding values of 0.03 as can be seen in Fig. 4.

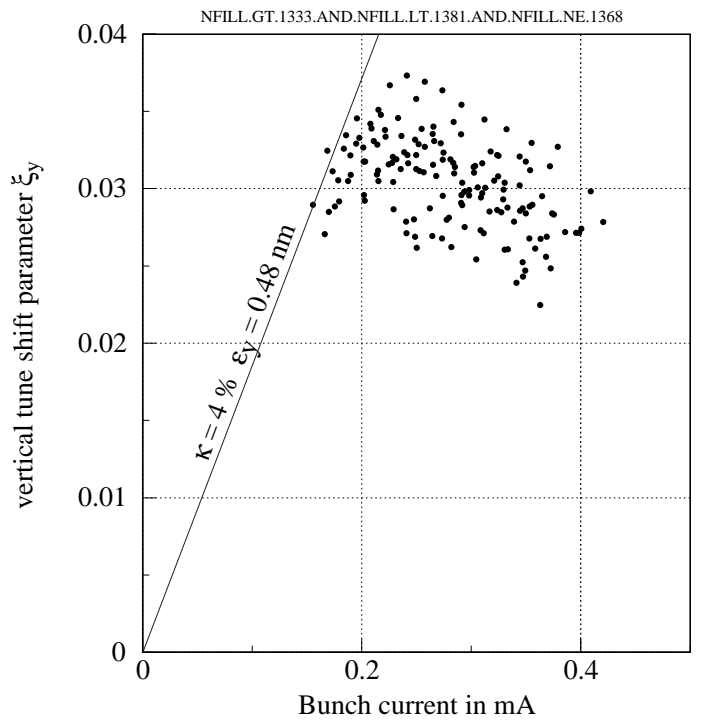

Figure 4: Vertical beam-beam tune shift parameter $\xi_{y}$ in 1992 for $4+4$ bunches,

LEP beams are colliding in four interaction points. Up to 1992 , LEP was filled with 4 electron and 4 positron 
bunches. Local vertical separation was used to avoid unwanted collisions. From 1993 on, regular operation was using $8+8$ bunches with horizontal separation in the arcs of LEP using the Pretzel scheme [2]. Residual vertical and horizontal separation was minimized and allowed beambeam tune shifts similar to the best $4+4$ operation. Still, fills with similar currents resulted in luminosities and vertical beam-beam tune shift parameters $\xi_{y}$ varying significantly $( \pm 30 \%)$. Vertical orbit corrections had significant effects on luminosity. However, it was not sufficient to reach a small rms in the vertical orbit. Correcting back to the particular structure of a vertical orbit saved in conditions with excellent beam-beam tune shift ("golden orbit") gave reproducibly good results. 1994 optics and running conditions were unchanged compared to 1993. Stable operation at one single beam energy and extensive use of reloading and reproducing "golden orbits" resulted in excellent and rather reproducible performance with vertical beam-beam tune shifts exceeding 0.04 on many occasions, as can be seen in Fig. 5. "Golden orbits" are obtained empirically starting from a well corrected machine. The vertical orbit is modified by many small local and global vertical corrections and the result on luminosity recorded. The ingredients of a "golden orbit" are not exactly known, but certainly include a minimization of the vertical dispersion at the interaction points and of the coupling between the horizontal and vertical planes.

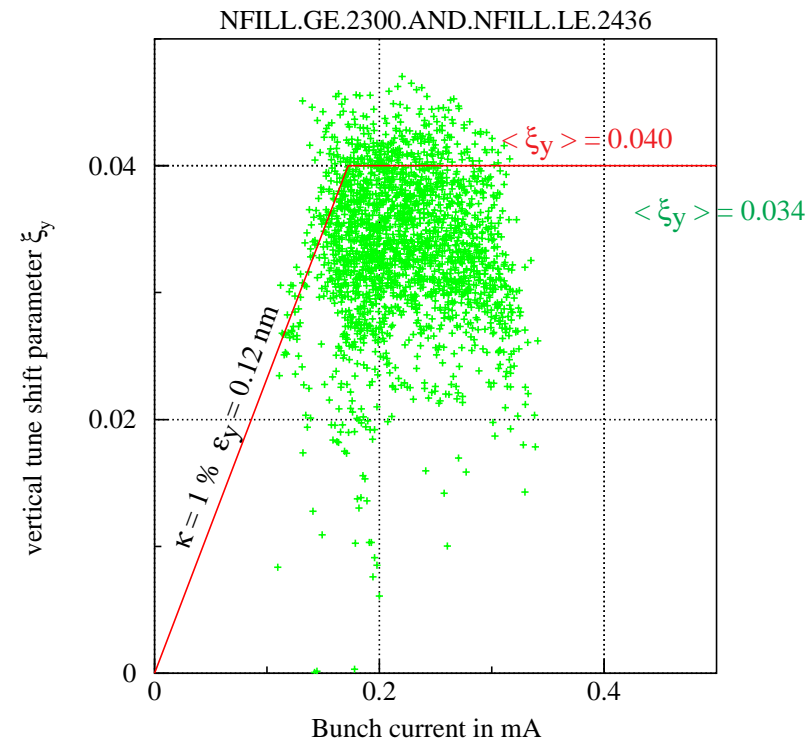

Figure 5: $\xi_{y}$ dependence on current in final Pretzel operation. The expected behavior for an emittance ratio of $\kappa=1 \%$ and a maximum $\xi_{y}=0.04$ is also shown.

Fig. 6 shows luminosity, currents, beam-beam tune shift and inverse beam-beam lifetimes for one of the best fills at LEP1 energies.

Beam lifetimes in LEP are well understood [3]. Single beam lifetimes are typically 45 hours at LEP1 energies and

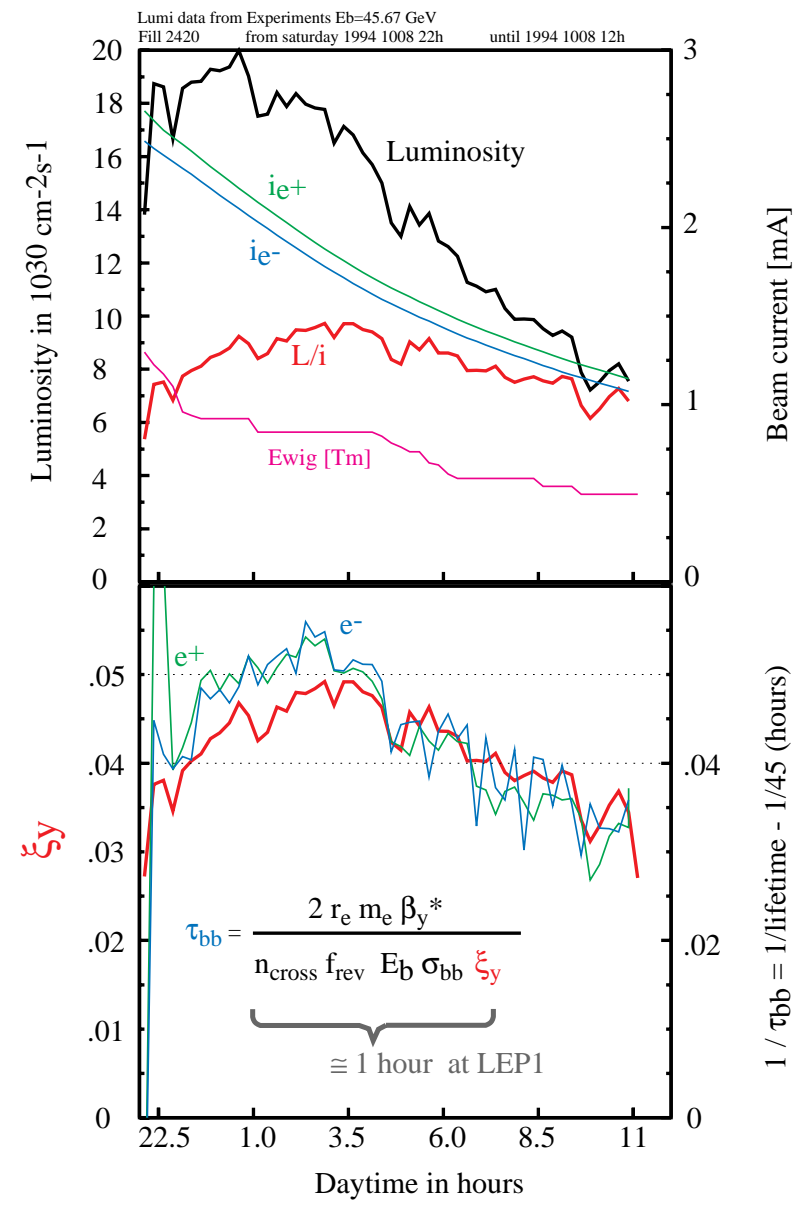

Figure 6: Luminosity, currents, beam-beam tune shift parameter $\xi_{y}$ and inverse beam-beam lifetimes of a very good fill at LEP1 with $8+8$ bunches in the Pretzel scheme.

mainly due to beam particles lost in Compton scattering on thermal photons, originating from the black body radiation of the beam pipe. In collisions, the lifetime decreases to typically 20 hours. The dominant process is radiative Bhabha scattering or beam-beam Bremsstrahlung. The lifetime in collisions $\tau_{b b}$ is inversely proportional to $\xi_{y}$, as shown in the lower part of Fig. 6. Extra losses at high currents are explained by scraping of non-Gaussian tails. At a low level, non-Gaussian tails were always observed in LEP both in the horizontal and the vertical plane and even without collisions. They can be explained to a large extent by scattering processes and off-momentum particles as described in [4].

The amount of non-Gaussian tails can increase significantly for high currents and beam-beam tune shifts. The maximum useful current, that could safely be collided at LEP1 energies was in fact limited to about $0.4 \mathrm{~mA}$ per bunch by background and lifetime problems due to the presence of strong non-Gaussian tails, mainly in the vertical plane.

Differences in intensities between the various electron and positron bunches up to a level of about $10 \%$ did not cause any problems. 
The performance in terms of vertical beam-beam tune shift decreased in 1995 when $12+12$ bunches were collided using bunch trains with vertical separation. This was attributed to residual vertical separation [5].

\section{LEP1.5, E=65 GEV}

LEP1 operation was stopped in october 1995. More superconducting radio frequency cavities were installed. This allowed in November 1995 to raise the beam energy to $65 \mathrm{GeV}$. The same optics with with $90^{\circ} / 60^{\circ}$ phase advance was used as previously at LEP1 energies. The natural horizontal emittance at $65 \mathrm{GeV}$ is then $\varepsilon_{x}=24 \mathrm{~nm}$. New record levels in beam-beam tune shift of $\xi_{y}=0.05$ and peak luminosities of $L=2.6 \cdot 10^{31} \mathrm{~cm}^{-2} \mathrm{~s}^{-1}$ with only $4+4$ bunches were obtained within 10 days of operation at this new energy.

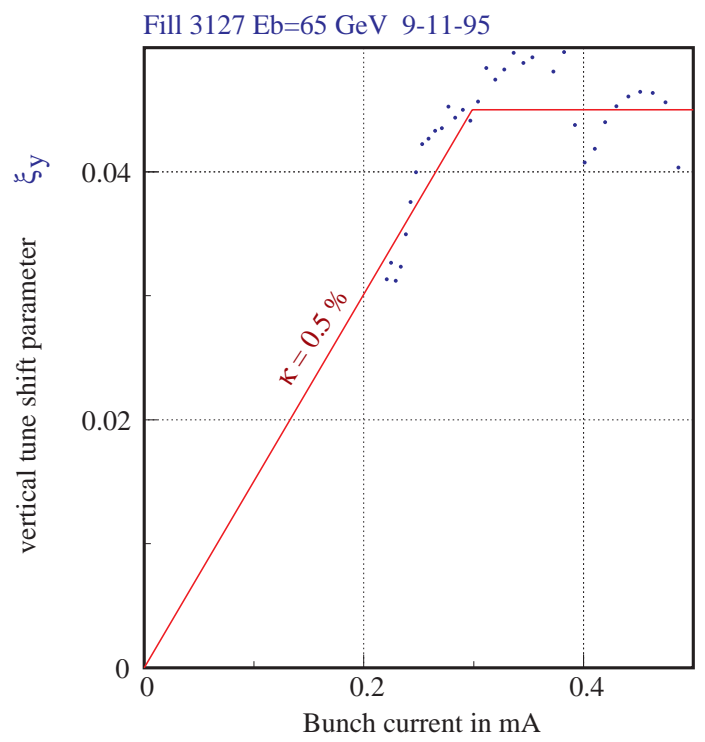

Figure 7: $\xi_{y}$ dependence on bunch current observed at a beam energy of $65 \mathrm{GeV}$. The data is shown as dots and the expected behavior for an emittance ratio $\kappa=0.5 \%$ and a maximum $\xi_{y}=0.045$ as line.

Fig. 7 shows the vertical beam-beam tune shift for a fill at $65 \mathrm{GeV}$. If we extrapolate the straight line in Fig. 7 we obtain an unperturbed vertical beam-beam tune shift of about 0.08 at a current of $0.5 \mathrm{~mA}$. There were no lifetime nor background problems when currents of $0.5 \mathrm{~mA}$ were collided at $65 \mathrm{GeV}$. The size of both beams increased symmetrically and there was no need for emittance control at this energy. It was possible to close collimators relatively tight without lifetime problems. In spite of the higher currents per bunch, there were less non Gaussian beam tails than at LEP1 energies [6].

\section{LEP2, E=86 GEV}

After adding many more superconducting cavitities in the shutdown between 1995 and 1996 and in a short stop in summer 1996, it was possible to raise the beam energy to
$86 \mathrm{GeV}$ [7]. The natural horizontal emittance at this energy is $\varepsilon_{x}=42 \mathrm{~nm}$ for the $90^{\circ} / 60^{\circ}$ lattice.

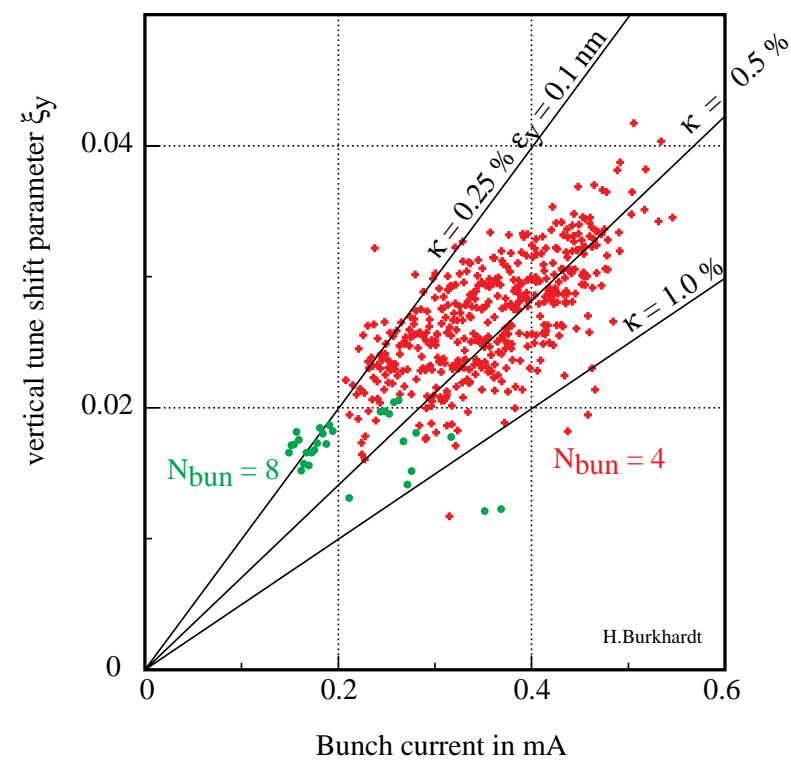

Figure 8: $90^{\circ} / 60^{\circ}$ optics, $\varepsilon_{x}=42 \mathrm{~nm}, 86 \mathrm{GeV}$

Fig. 8 shows the observed dependence of vertical beambeam tune shift on current. Vertical tune shifts exceeded 0.04 at the highest bunch currents that were available without any sign of saturation. The straight lines show the expected behavior for several emittance ratios. Vertical emittances down to $0.1 \mathrm{~nm}$ corresponding to an emittance ratio of only $0.25 \%$ were obtained in some cases.

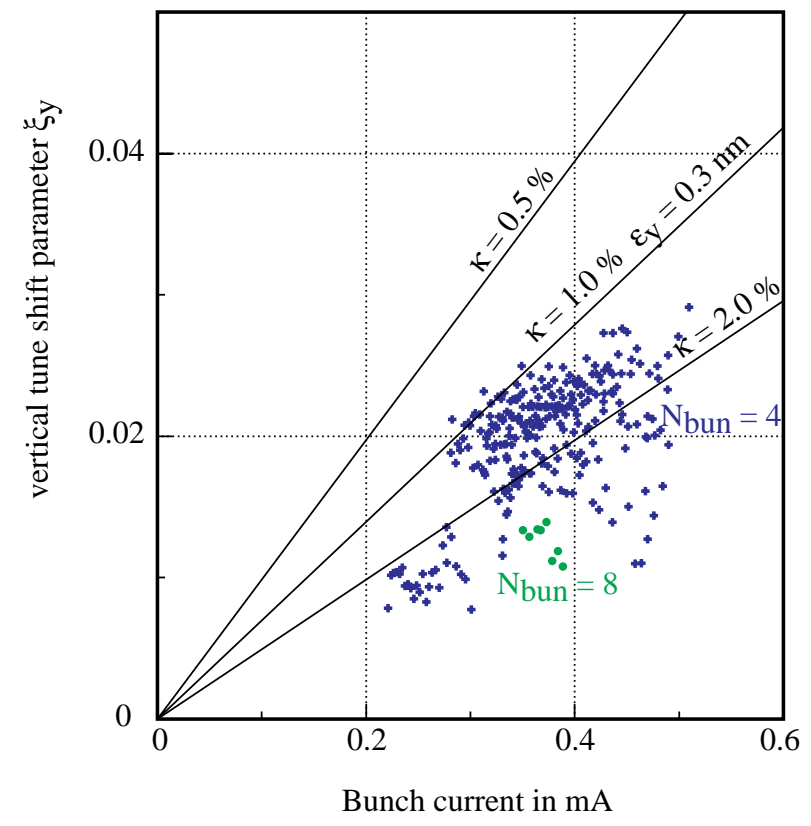

Figure 9: $108^{\circ} / 90^{\circ}$ optics, $\varepsilon_{x}=30 \mathrm{~nm}, 86 \mathrm{GeV}$

There was an attempt to decrease the horizontal emittance through an increase in the horizontal phase advance: An optics with $108^{\circ} / 90^{\circ}$ phase advance and a natural horizontal emittance of $\varepsilon_{x}=30 \mathrm{~nm}$ at $86 \mathrm{GeV}$ was used for 
the last three weeks of operation in 1996. Fig. 9 shows the observed vertical beam-beam tune shifts as function of current. The vertical emittance was larger than previously and overall performance did not improve [8].

Plans for 1997 are to return for regular operation to the well known $90^{\circ} / 60^{\circ}$ optics and to attempt to reduce the horizontal emittance by an increase in the horizontal damping partition to a value of $J_{x} \approx 1.5$. The impedance of LEP will be decreased by the removal of normal conducting cavities and it should be possible to increase the current per bunch. Since there were no signs yet of a saturation in the vertical beam-beam tune shift at LEP2 energies, we hope this year to reach new record levels of maybe $\xi_{y}=0.06$.

\section{SUMMARY, CONCLUSION}

We described observations of beam-beam interactions in LEP, based on 7 years of operation, covering about a factor of two in beam energies from 45 to $86 \mathrm{GeV}$.

Beam lifetimes are well understood at all energies in LEP and accounted for by particle scattering processes.

The vertical beam-beam tune shift parameter $\xi_{y}$ exceeded 0.04 at all energies. Good performance needs a well corrected machine and careful operational tuning (golden orbit).

The main observations at the three energies can be summarized as follows:

LEP1, 45.6 GeV : Using an optics with $90^{\circ}$ phase advance in the horizontal plane and a wiggler placed in a region with dispersion, it was possible to adjust the horizontal emittance between 12 and $36 \mathrm{~nm}$, and to operate safely at the beam-beam limit in fills lasting over 10 hours. The maximum useful current in collision was limited to about $0.4 \mathrm{~mA}$ by non-Gaussian transverse beam tails, generating background to the experiments and reducing the beam lifetime.

LEP1.5, $65 \mathrm{GeV}$ : Going from 45.6 to $65 \mathrm{GeV}$ implied an increase of the horizontal emittance from 12 to $24 \mathrm{~nm}$ and a reduction of the damping times by nearly a factor of three. Operation was very stable, there were less non-Gaussian tails and no problem to collide currents of $0.5 \mathrm{~mA}$. Vertical beam-beam tune shifts up to about 0.05 were reached after only 10 days of operation at the new energy.

LEP2, $86 \mathrm{GeV}$ : Most of the operation at this energy was based on the same optics with $90^{\circ}$ phase advance in the horizontal plane as used at lower energies. The natural horizontal emittance at this energy is $42 \mathrm{~nm}$. Vertical beam-beam tune shifts increased linearly with current, reaching 0.04 without any signs of saturation. An attempt to improve performance using the higher phase advance of $108^{\circ}$ in the horizontal plane was not so successful. Plans for this year are to return to the $90^{\circ}$ phase advance optics and to reduce the horizontal emittance by an increase of the horizontal damping partition.

\section{REFERENCES}

[1] G. Arduini et al. LEP1 Operation, 1989-1995. In V. Suller and Ch. Petit-Jean-Genaz, editors, Proceedings of the 5th European Particle Accelerator Conference, volume 1, pages 286-288, 1996.

[2] R. Bailey et al. LEP Operation in 1993 with the Pretzel Scheme. In V. Suller and Ch. Petit-Jean-Genaz, editors, Proceedings of the 4th European Particle Accelerator Conference, volume I, pages 439-441, 1994.

[3] H. Burkhardt and R. Kleiss. Beam Lifetimes in LEP. In V. Suller and Ch. Petit-Jean-Genaz, editors, Proceedings of the 4th European Particle Accelerator Conference, volume II, pages 1353-1355, 1994.

[4] I. Reichel, H. Burkhardt and G. Roy. Observation and Simulation of Beam Tails in LEP. These proceedings.

[5] W. Herr et al. Experience with Bunch Trains in LEP. These proceedings.

[6] I. Reichel. The Loss Monitors at High Energy. In J. Poole, editor, Proceedings of the 6th Workshop on LEP Performance, pages 137-140, 1996.

[7] S. Myers. LEP2: Present and Future Performance and Limitations. These proceedings.

[8] D. Brandt et al. Low Emittance Lattices for LEP2. These proceedings. 